\title{
Familial, Cognitive, and Behavioral Characteristics of Adolescents with Depression
}

\author{
Yeeun Lee ${ }^{1}$, Bung-Nyun Kim², Min-Hyeon Park ${ }^{3}$, and Subin Park ${ }^{4}$ \\ ${ }^{1}$ Department of Psychology, Korea University, Seoul, Korea \\ ${ }^{2}$ Department of Psychiatry and Behavioral Science, Seoul National University College of Medicine, Seoul, Korea \\ ${ }^{3}$ Department of Psychiatry, The Catholic University of Korea, St. Paul's Hospital, Seoul, Korea \\ ${ }^{4}$ Department of Research Planning, Mental Health Research Institute, National Center for Mental Health, Seoul, Korea
}

\begin{abstract}
Objectives: Adolescent depression is a complex disorder influenced by a variety of personal and familial factors. In this study, we compared the familial, cognitive, and behavioral characteristics of adolescents with and without diagnosed depression.

Methods: Forty adolescents with depressive disorder were recruited from two psychiatric clinics, along with 46 healthy adolescents from a middle school and a high school. We then compared the participants' cognitive and behavioral characteristics and the childrearing attitudes of their parents.

Results: Compared to the healthy adolescents, the adolescents with depression exhibited lower self-esteem, higher emotional reappraisal, greater disruptive behavior, and lower attention. Furthermore, compared to the mothers of the healthy adolescents, the mothers of those with depression reported less affective, less autonomic, and more rejecting parenting attitudes towards their children.

Conclusion: We found that attentional problems, negative parenting attitudes, negative self-cognition, and expressive suppression are all associated with adolescent depression. Parenting education and interventions appear to be needed to correct the negative cognitions of adolescents with depression.
\end{abstract}

Key Words: Depression; Adolescent; Parenting.

Received: March 18, 2017 / Revision: May 15, 2017 / Accepted: May 31, 2017

Address for correspondence: Subin Park, Department of Research Planning, Mental Health Research Institute, National Center for Mental Health, 127 Yongmasan-ro, Gwangin-gu, Seoul 04933, Korea

Tel: +82-2-2204-0108, Fax: +82-2-2204-0280, E-mail: subin-21@hanmail.net

\section{INTRODUCTION}

Depression is a prevalent disease among adolescents: roughly $12.5 \%$ of the adolescent population in the U.S. have reported experiencing at least one major depressive episode in the past year, ${ }^{1)}$ while $7-19 \%$ of adolescents had a diagnosis of depression across 11 European countries. ${ }^{2)}$ Depression is one of the top-five causes of morbidity in the adolescent population. ${ }^{3)}$ It can impair social functioning and academic performance, and is often accompanied by other psychological and behavioral problems, such as substance abuse and disruptive behavior disorders. ${ }^{4)}$ Furthermore, suicide is the third leading cause of death in this population, ${ }^{3)}$ which suggests that the heightened risk of suicidal behaviors among adolescents with depression warrants further attention. ${ }^{5}$

Despite the high prevalence of depression among adolescents and its potential adverse outcomes, depressive symp-

This is an Open Access article distributed under the terms of the Creative Commons Attribution Non-Commercial License (http://creativecommons.org/licenses/by-nc/4.0) which permits unrestricted non-commercial use, distribution, and reproduction in any medium, provided the original work is properly cited. toms are often under-recognized. They are often attributed to "growing pains"-namely, the normal stress of adolescenceor misdiagnosed as conduct and behavioral problems. ${ }^{4)}$ For instance, Sund et al., ${ }^{6}$ using national data from Norway, found that less than $20 \%$ of currently depressed adolescents had utilized professional services to manage their emotional difficulties, suggesting that a high proportion of depressed adolescents go untreated. Untreated adolescent depression can have a life-long impact: adolescents with major depressive disorder (MDD) are more likely to have depression and other mental disorders in adulthood ${ }^{7}$ and even sub-threshold depression is associated with an increased risk of future $\mathrm{MDD}^{8}{ }^{8}$ With adolescence being a peak in the prevalence and incidence of depression, ${ }^{4}$ it is a critical point for early intervention and prevention of life-long depression.

The prevalence of depression among Korean adolescents is particularly high. Cho et al." showed that $34.3 \%$ to $47.5 \%$ of Korean adolescents living in an urban area reported probable depressive symptoms [using a Center for Epidemiological Studies Depression Scale (CES-D) score of 16 as a cutoff], 
while $17.4 \%$ to $20.6 \%$ reported severe depressive symptoms (using a CES-D score of 25 as a cutoff). To prevent an epidemic of adolescent depression, high-risk groups must be identified and early intervention provided. Previous studies have accumulated evidence on both environmental and personal factors associated with an increased risk of adolescent depression, including parental maltreatment, negative self-views, and poor coping style. ${ }^{10-12)}$ To determine the distinctive features of Korean adolescents with depression, we compared the parental, cognitive, and behavioral characteristics of depressed adolescents with those of healthy adolescents in a Korean adolescent sample.

\section{METHODS}

\section{Subjects and procedures}

Forty psychiatric outpatients aged $12-17$ years with a primary diagnosis of depressive disorder based on the Diagnostic and Statistical Manual of Mental Disorders, 4th edition, Text Revision (DSM-IV-TR) were enrolled in this study by child psychiatrists in two general hospitals in Seoul, Republic of Korea. These psychiatric diagnoses were confirmed using the Korean Kiddie Schedule for Affective Disorders and Schizophrenia-Present and Lifetime version (K-SADS-PL). ${ }^{13)}$ The exclusion criteria were as follows: 1) having a hereditary genetic disorder; 2) having a current/past history of brain trauma, organic brain disorder, seizure, or any neurological disorder; and 3) having been diagnosed with a pervasive developmental disorder or mental retardation. Participants' intelligence quotient (IQ) was calculated using the abbreviated form of the Korean Educational Development Institute-Wechsler Intelligence Scale for Children. ${ }^{14)}$ We also recruited 46 healthy adolescents aged 12-17 years as a comparison group from a middle school and a high school near our research center. All of them were evaluated by a child psychiatrist and screened for depression and other psychiatric disorders using the Korean version of the K-SADS-PL. The exclusion criteria for the healthy controls were the same as those for the depression group, with the added criterion that they could not have depression, bipolar disorder, schizophrenia, or any other psychotic disorder. This study was approved by the Institutional Review Board for human subjects at the National Center for Mental Health (No. 2014-34) and the Seoul National University Hospital (No. C-1412-081-633). The data were collected over a year.

\section{Measures}

\author{
The Children's Depression Inventory (CDI) \\ The Children's Depression Inventory (CDI) is a self-rated,
}

symptom-oriented scale for youth aged 7-17 years. It comprises 27 items rated on a 3-point Likert Scale ranging from 0 (not present) to 2 (present and marked); total scores range from 0 to 54 . This scale assesses quantifiable symptoms such as depressed mood, hedonic capacity, vegetative symptoms, negative self-evaluation, and interpersonal problems (Cronbach's alpha $=0.82$ ). Participants were asked to complete the Korean version of the CDI. ${ }^{15)}$

\section{The Maternal Behavior Research Instrument (MBRI)}

The Maternal Behavior Research Instrument (MBRI) is a 48-item, self-report instrument assessing maternal parenting attitudes, which can be classified as affective (e.g., "It is fun to spend time with my child"), rejecting (e.g., "I ignore my child's demands"), autonomic (e.g., "I let my child do his/her own thing alone"), and controlling (e.g., "Children should by all means be obedient to their parents"). Each parenting attitude subscale comprises 12 items, and each item is rated on a scale of 1 to 5 ; the subscale scores, which range from $12-60$, are determined by summing the scores of the 12 constituent items. Higher scores on each subscale indicate that mothers more strongly endorse that attitude. The mothers of participants were asked to complete the Korean version of the MBRI. ${ }^{16)}$

\section{The Rosenberg Self-Esteem Scale (RSES)}

The Rosenberg Self-Esteem Scale (RSES) is a widely used self-report instrument for assessing global self-worth. This scale contains ten items (e.g., I feel that I have a number of good qualities, Cronbach's alpha $=0.80$ ), each of which is rated on a 5-point Likert Scale, ranging from 1 (strongly disagree) to 5 (strongly agree). The total Score ranges from 10 to 50 , with higher scores indicating higher self-esteem. Participants were asked to complete the Korean version of the RSES. ${ }^{17)}$

\section{Emotion Regulation Questionnaire (ERQ)}

The Emotion Regulation Questionnaire (ERQ) is a ten-item self-report scale assessing two specific emotion regulation strategies: 1) cognitive reappraisal (e.g., "When I'm faced with a stressful situation, I make myself think about it in a way that helps me stay calm") and 2) expressive suppression (e.g., "I control my emotions by not expressing them"). The respondents answer each item on a 7-point Likert Scale ranging from 1 (strongly disagree) to 7 (strongly agree). Higher scores on each subscale indicate that the respondent uses the corresponding emotion regulation strategy more frequently. Participants were asked to complete the Korean version of the ERQ. ${ }^{18,19)}$

\section{Disruptive Behavioral Disorder Scale (DBDS)}

The Disruptive Behavioral Disorder Scale (DBDS) is a par- 
ent-rated scale that comprises 8 items corresponding to the DSM-IV criteria for oppositional defiant disorders (ODD) and 15 items corresponding to the DSM-IV criteria for conduct disorder. The DBDS items are rated on a four-point Likert Scale corresponding to the severity of symptoms. Higher scores indicate more severe disruptive behavioral problems. The mothers of participants were asked to complete the Korean version of the DBDS. ${ }^{20,21)}$

\section{Stroop Color Word Interference Test (Stroop test)}

The Stroop test assesses cognitive functioning, including attention deployment, cognitive flexibility, and resistance to interference from outside stimuli. ${ }^{22)}$ The Stroop test requires respondents to first read the words "red," "green," and "blue" (Word subtest), after which they must state the colors of the letters of these words; the letter colors are either concordant (Color subtest) or discordant (Color-Word subtest) with the color words. An interference score is calculated by subtracting the number of correctly stated discordant-colored letters (i.e., the Color-Word score) from the number of correctly stated concordant-colored letters (Color score). All data are presented as t-scores adjusted for age and sex. Higher t-scores indicate better test performance.

\section{Statistical analyses}

We compared demographic characteristics, mother's parenting style, and cognitive and behavioral characteristics between children with and without depression using an independent t-test for continuous variables and a chi-squared test for categorical variables. We then conducted regression analyses to examine the relationships between CDI scores and the various familial or behavioral characteristics; participants' age was included as a covariate because it was significantly and inversely correlated with CDI scores $(\mathrm{r}=-0.38, \mathrm{p}<0.001)$. SPSS Statistics 21.0 (IBM Corp., Armonk, NY, USA) was used to perform all statistical analyses, and an alpha of less than 0.05 was considered significant.

\section{RESULTS}

The depression group comprised adolescents diagnosed with MDD (37.5\%), depressive disorder not otherwise specified (57.5\%), and MDD with dysthymia (5\%). The CDI scores were significantly higher in adolescents with depression than in healthy adolescents. There were no significant differences in age, sex, family income, parental education years, or IQ scores between the groups. However, adolescents with depression had a higher prevalence of other psychiatric disorders in comparison with healthy adolescents, including attentiondeficit hyperactivity disorder (ADHD) and anxiety disorder (Table 1).

Mothers of adolescents with depression reported less affective ( $\mathrm{p}=0.002)$, more rejecting $(\mathrm{p}=0.021)$, and less autonomic $(\mathrm{p}=0.006)$ parenting attitudes than did the mothers of healthy adolescents. However, the groups showed no significant difference in controlling parenting attitudes (Table 2).

Adolescents with depression had lower self-esteem than did healthy adolescents $(p<0.001)$. Concerning the emotional regulation strategies, adolescents with depression reported using expressive suppression more than did healthy adolescents $(\mathrm{p}=0.021)$, but the groups did not significantly differ in cognitive reappraisal ( $\mathrm{p}=0.164)$. Among disruptive behavioral disorders, parent-rated scores for ODD were higher among adolescents with depression than among healthy adolescents

Table 1. Characteristics of participants

\begin{tabular}{|c|c|c|c|c|}
\hline Characteristics & Control $(n=46)$ & Depression $(n=40)$ & $t / \chi^{2}$ & $\mathrm{p}$ \\
\hline Age, mean (SD) & $14.76(1.35)$ & $14.20(1.30)$ & 1.95 & 0.055 \\
\hline Sex, male, \% & 58.7 & 65.0 & 0.36 & 0.549 \\
\hline Family income (won), \% & & & 1.33 & 0.515 \\
\hline$\leq 2,000,000$ & 20.0 & 25.0 & & \\
\hline $2,010,000-4,000,000$ & 28.0 & 40.0 & & \\
\hline$>4,000,000$ & 52.0 & 35.0 & & \\
\hline Paternal educational years, mean (SD) & $13.78(2.83)$ & $14.72(2.17)$ & 1.30 & 0.201 \\
\hline Maternal educational years, mean (SD) & $13.67(2.96)$ & $13.62(2.38)$ & 0.07 & 0.945 \\
\hline CDI score, mean (SD) & $13.60(5.79)$ & $19.88(6.51)$ & -4.71 & $<0.001$ \\
\hline IQ, mean (SD) & $100.68(14.70)$ & $106.41(14.03)$ & -1.77 & 0.080 \\
\hline Comorbidity, n (\%) & $5(10.9)$ & $15(37.5)$ & 8.50 & 0.005 \\
\hline Anxiety disorder & $3(6.5)$ & $5(12.5)$ & & \\
\hline ADHD & $0(0)$ & $11(27.5)$ & & \\
\hline Tic disorder & $2(4.3)$ & $0(0)$ & & \\
\hline
\end{tabular}

ADHD: attention-deficit hyperactivity disorder, CDI: Children's Depression Inventory, IQ: intelligence quotient 
Table 2. Comparison of parenting styles

\begin{tabular}{|c|c|c|c|c|}
\hline \multirow{2}{*}{ Parenting style } & Control $(n=46)$ & Depression $(n=40)$ & \multirow{2}{*}{$\dagger$} & \multirow{2}{*}{$\mathrm{p}$} \\
\hline & Mean (SD) & Mean $(S D)$ & & \\
\hline Affective parenting & $46.18(7.55)$ & $40.39(6.77)$ & 3.28 & 0.002 \\
\hline Rejecting parenting & $30.30(6.19)$ & $33.94(6.33)$ & -2.36 & 0.021 \\
\hline Autonomic parenting & $43.58(3.82)$ & $40.30(5.45)$ & 2.83 & 0.006 \\
\hline Controlling parenting & $40.61(4.81)$ & $40.97(4.88)$ & -0.31 & 0.761 \\
\hline
\end{tabular}

Table 3. Comparison of cognitive and behavioral characteristics

\begin{tabular}{|c|c|c|c|c|}
\hline \multirow{2}{*}{ Characteristics } & Control $(n=46)$ & Depression $(n=40)$ & \multirow{2}{*}{$\dagger$} & \multirow{2}{*}{$\mathrm{p}$} \\
\hline & Mean (SD) & Mean (SD) & & \\
\hline Rosenberg Self-Esteem Scale & $31.33(6.39)$ & $26.00(4.47)$ & 4.29 & $<0.001$ \\
\hline \multicolumn{5}{|c|}{ Emotional Regulation Questionnaire } \\
\hline Cognitive reappraisal & $20.98(4.94)$ & $19.50(4.53)$ & 1.40 & 0.164 \\
\hline Expressive suppression & $9.82(2.71)$ & $11.32(3.07)$ & -2.35 & 0.021 \\
\hline \multicolumn{5}{|c|}{ Disruptive Behavioral Disorder Scale } \\
\hline Oppositional defiant disorder & $1.53(2.25)$ & $5.08(5.12)$ & -3.99 & $<0.001$ \\
\hline Conduct disorder & $0.49(1.12)$ & $1.28(3.65)$ & -1.36 & 0.178 \\
\hline \multicolumn{5}{|l|}{ Stroop } \\
\hline Word & $52.13(10.06)$ & $45.10(9.73)$ & 3.27 & 0.002 \\
\hline Color & $48.15(9.95)$ & $47.18(10.63)$ & 0.43 & 0.672 \\
\hline Color-Word & $48.34(11.21)$ & $48.85(10.31)$ & -0.25 & 0.800 \\
\hline Interference & $51.66(9.41)$ & $53.95(12.32)$ & -0.94 & 0.349 \\
\hline
\end{tabular}

Table 4. Relationships between depressive symptoms and cognitive or behavioral characteristics $(n=86)$

\begin{tabular}{lcc}
\hline \multirow{2}{*}{$\begin{array}{c}\text { Familial/behavioral } \\
\text { characteristics }\end{array}$} & \multicolumn{2}{c}{ Children's Depression Inventory } \\
\cline { 2 - 3 } & $\mathrm{B}(95 \% \mathrm{Cl})^{*}$ & $\mathrm{p}$ \\
\hline Rosenberg Self-Esteem Scale & $-0.35(-0.57,-0.13)$ & 0.002 \\
Cognitive reappraisal & $-0.37(-0.67,-0.08)$ & 0.014 \\
Expressive suppression & $0.51(0.04,0.99)$ & 0.035 \\
Oppositional defiant disorder & $0.18(-0.16,0.51)$ & 0.298 \\
Conduct disorder & $0.18(-0.36,0.71)$ & 0.517 \\
Stroop Word & $-0.09(-0.22,0.05)$ & 0.198 \\
Stroop Color & $0.01(-0.12 .0 .15)$ & 0.878 \\
Stroop Color-Word & $0.01(-0.12,0.14)$ & 0.840 \\
Stroop Interference & $0.06(-0.07,0.18)$ & 0.363 \\
\hline
\end{tabular}

*unstandardized beta coefficients adjusted for age

$(\mathrm{p}<0.001)$. There were no significant differences in scores for conduct disorder between the two groups $(\mathrm{p}=0.178)$. On the Stroop test, adolescents with depression had a lower score for the Word subtest than did healthy adolescents, but the groups did not show a significant difference in the other test scores (Table 3).

In the total sample, the CDI scores were negatively associated with self-esteem $(B=-0.35, p=0.002)$ and cognitive reappraisal $(B=-0.37, p=0.014)$, and positively associated with expressive suppression $(B=0.51, p=0.035)$, after controlling for participants' age (Table 4).

\section{DISCUSSION}

In the present study, we examined the distinct parental, cognitive, and behavioral characteristics of depressed and healthy adolescents. We found significant differences between these two groups in maternal parenting attitudes, subjective evaluations of self-worth, emotional regulation strategies, disruptive behavioral symptoms, and cognitive functioning.

The mothers of adolescents with depression tended to be less emotionally supportive, give less autonomy to their children, and be more likely to ignore children's needs than were the mothers of healthy adolescents. These findings are consistent with those of prior studies showing that family climate and parenting style have strong impacts on the development of depression in children and adolescents. ${ }^{12,23)}$ Specifically, our findings support the notion that maternal acceptance and warmth are associated with decreased risk of adolescent depression, while maternal hostility increases the risk. ${ }^{23)}$ Additionally, in their meta-analysis, Yap et al. ${ }^{12)}$ noted that parental over-involvement (i.e., interference with children's autonomy) and a lack of granting autonomy increased the risk of adolescent depression, which suggests that less autonomic parenting attitudes among mothers are also a risk factor for depressive symptoms.

Consistent with prior studies showing a strong association between low self-esteem and depressive symptoms (for a re- 
view $^{24)}$ ), adolescents with depression had lower self-esteem than did healthy adolescents, and all participants' self-esteem scores were inversely correlated with their level of depressive symptoms. To identify the nature of the relationship between self-esteem and depression, Sowislo and Orth $^{24)}$ meta-analyzed existing longitudinal data on these two variables. They found that the influence of self-esteem on depression was consistent and robust, and was stronger than the effect of depression on self-esteem. This supports the vulnerability model, which posits that negative self-perceptions might be a causal risk factor for the development and maintenance of depression. ${ }^{25)}$ In line with these longitudinal findings, our results suggest that lower self-esteem might put adolescents at risk of depressive symptoms.

Adolescents with depression had a greater tendency to suppress their emotional expression than did healthy adolescents. Furthermore, use of expressive suppression and cognitive reappraisal were positively and negatively associated, respectively, with the extent of depressive symptoms. The association between expressive suppression and depression accords with prior findings on the adverse effect of emotion suppression on various psychological and social outcomes. ${ }^{18,26)}$ In contrast to cognitive appraisal, which is associated with beneficial changes in positive and negative emotions, people who employ expressive suppression tend to experience more negative emotions and less positive emotions, and overall have poorer psychological well-being than do others; this has been confirmed in both cross-sectional and experimental studies. ${ }^{18,27)}$ This ineffectiveness of suppressing emotions, even negative ones, might explain the association between depressed adolescents' tendency to engage in such suppression and their affective symptoms. The significant role of emotion regulation in the mood symptoms of adolescent ${ }^{28)}$ suggests that they might benefit from psychological interventions focusing on emotion regulation, especially emotion expression.

The parents of adolescents with depression rated adolescents as having more severe disruptive behavioral symptoms in comparison to healthy adolescents, particularly ODD symptoms. We pose two possible explanations for this. First, adolescents with depression might be more likely to have comorbid disruptive disorders when compared with healthy adolescents. Disruptive disorders are one of the most common comorbid psychiatric diagnoses of depression. ${ }^{29)}$ More specifically, a longitudinal study by Kovacs et al. ${ }^{30}$ indicated that conduct problems can develop as a complication of depressive symptoms. Such comorbidities have been shown to predict distinct prognoses in adolescents, such as a lower relapse rate of depression but a higher risk of suicide attempts and worse short-term outcomes; ${ }^{29)}$ thus, adolescents with depression who have comorbid disruptive behavioral symp- toms may need special attention. Second, it is possible that the parents of depressed adolescents perceive adolescents' depressive symptoms as behavioral problems. For instance, diminished attentional ability and irritability, which are frequent symptoms of adolescent depression, ${ }^{31)}$ can be perceived by others as indicative of behavioral problems such as ODD. To further understand the mechanism of the association between depressive symptoms and disruptive behavioral problems in adolescents, further studies are needed.

Adolescents with depression showed poorer performance on the Stroop Word subtest than did healthy adolescents, but not in the other subtests. These findings suggest that adolescents with depression show diminished cognitive performance for a relatively automatic and less cognitively demanding task. Our results are consistent with Den Hartog et al., ${ }^{32)}$ who found that patients with depression show cognitive deficits in an autonomic processing subtask (e.g., the Word and Color subtests), but intact performance for a more effortful cognitive task (e.g., the interference subtest). These findings support the cognitive speed hypothesis, ${ }^{32)}$ which posits that the cognitive dysfunction evident in patients with depression are due to their reduced speed of autonomic information processing, rather than impaired performance on effortful tasks. Our findings similarly support the notion that adolescents with depression show worse cognitive speed in autonomic tasks, which might underlie their deficits in overall cognitive functioning.

One limitation of this study is the small sample size, which might have reduced the statistical power. Another limitation is that the different cognitive and behavioral characteristics of these two groups might be attributable to factors other than depression, such as the comorbid disorders. For instance, the relatively lower performance on the Stroop Word subtest or the increased tendency for disruptive behaviors in adolescents with depression might be associated with the higher prevalence of ADHD in that group. Finally, this study was cross-sectional, and thus the data cannot offer information on the causality of the relationships between variables. For instance, while adolescents' expressive suppression might contribute to the development and maintenance of depressive symptoms, depressive symptoms might also inhibit their emotional expression. Thus, to confirm the current findings and understand the causal relationships between each variable and depressive symptoms in adolescents, further longitudinal studies using a larger sample are needed.

\section{CONCLUSION}

In this pilot study, we identified distinct familial and personal characteristics of adolescents with depression in com- 
parison to healthy adolescents. More specifically, adolescents with depression tended to receive less affective and autonomic parenting from their mothers, have more negative beliefs about themselves, suppress their authentic emotions, have difficulty in cognitive tasks, and present behavioral problems. Although our findings cannot confirm the causal relationships between these characteristics and depressive symptoms, the knowledge that adolescents with depression might experience a wide range of difficulties in multiple domains indicates the need for an integrative intervention approach. In particular, considering the associations of depression with negative parenting, negative self-cognitions, and expressive suppression, parenting education and interventions aimed at correcting negative cognitions are needed for adolescents with depression.

\section{Acknowledgments}

This work was supported by the National Research Foundation of Korea (NRF) Grant funded by the Korean Government (MSIP) (NRF2014R1A1A3049818).

\section{Conflicts of Interest}

The authors have no financial conflicts of interest.

\section{REFERENCES}

1) Major Depression Among Adolescents [cited $2017 \mathrm{Feb} 23]$. Available from: https://www.nimh.nih.gov/health/statistics/prevalence/majordepression-among-adolescents.shtml.

2) Balazs J, Miklósi M, Keresztény Á, Apter A, Bobes J, Brunner R, et al. P-259 - prevalence of adolescent depression in Europe. Eur Psychiatry 2012;27:1.

3) Adolescent health epidemiology [cited $2017 \mathrm{Feb} 23$ ]. Available from: http://www.who.int/maternal_child_adolescent/epidemiology/adolescence/en/.

4) Weller EB, Weller RA. Depression in adolescents growing pains or true morbidity? J Affect Disord 2000;61 Suppl 1:9-13.

5) Thapar A, Collishaw S, Pine DS, Thapar AK. Depression in adolescence. Lancet 2012;379:1056-1067.

6) Sund AM, Larsson B, Wichstrøm L. Prevalence and characteristics of depressive disorders in early adolescents in central Norway. Child Adolesc Psychiatry Ment Health 2011;5:28.

7) Lewinsohn PM, Rohde P, Klein DN, Seeley JR. Natural course of adolescent major depressive disorder: I. Continuity into young adulthood. J Am Acad Child Adolesc Psychiatry 1999;38:56-63.

8) Hill RM, Pettit JW, Lewinsohn PM, Seeley JR, Klein DN. Escalation to major depressive disorder among adolescents with subthreshold depressive symptoms: evidence of distinct subgroups at risk. J Affect Disord 2014;158:133-138.

9) Cho SJ, Jeon HJ, Kim MJ, Kim JK, Kim US, Lyoo IK, et al. Prevalence and correlates of depressive symptoms among the adolescents in an urban area in Korea. J Korean Neuropsychiatr Assoc 2001;40: 627-639.

10) Robertson JF, Simons RL. Family factors, self-esteem, and adolescent depression. J Marriage Fam 1989;51:125-138.

11) Cairns KE, Yap MB, Pilkington PD, Jorm AF. Risk and protective factors for depression that adolescents can modify: a systematic review and meta-analysis of longitudinal studies. J Affect Disord 2014; 169:61-75.

12) Yap MB, Pilkington PD, Ryan SM, Jorm AF. Parental factors associated with depression and anxiety in young people: a systematic review and meta-analysis. J Affect Disord 2014;156:8-23.

13) Kim YS, Cheon KA, Kim BN, Chang SA, Yoo HJ, Kim JW, et al. The reliability and validity of Kiddie-Schedule for Affective Disorders and Schizophrenia-Present and Lifetime version- Korean version (K-SADS-PL-K). Yonsei Med J 2004;45:81-89.

14) Park KS, Yoon JY, Park HJ, Park HJ, Kwon KU. Development of KEDI-WISC, individual intelligence test for Korean children. Seoul: Korean Educational Development Institute;1996.

15) Cho SC, Yi YS. Development of the Korean form of the Kovacs' Childeren's Depression Inventory. J Korean Neuropsychiatr Assoc 1990;29:943-956.

16) Kim SJ, Kim YH, Kim KS. Mothers' child-rearing attitude. Korean J Child Health Nurs 2003;9:392-398.

17) Lee JY, Nam SK, Lee MK, Lee JH, Lee SM. The Rosenberg's self-esteem scale: a validation study. Korean J Couns Psychother 2009;21: 73-89.

18) Gross JJ, John OP. Individual differences in two emotion regulation processes: implications for affect, relationships, and well-being. J Pers Soc Psychol 2003;85:348-362.

19) Han SH, Hyun OK. Relationships of positive and negative emotion to cognitive reappraisal and expressive suppression emotional regulation strategies and self-control in adolescence. Korean J Child Stud 2006;27:1-11.

20) Pelham WE Jr, Gnagy EM, Greenslade KE, Milich R. Teacher ratings of DSM-III-R symptoms for the disruptive behavior disorders. J Am Acad Child Adolesc Psychiatry 1992;31:210-218.

21) Cho SC, Kim JW, Shin MS, Hwang JW, Han SW, Park KH, et al. Behavioral and emotional problems in children with nocturnal enuresis: results of a multicenter study. J Korean Neuropsychiatr Assoc 2005;44:730-735.

22) Shin MS, Park MJ. A standardization study for Korean version of the Stroop Color-Word Test children's version. Seoul: The Korean Psychological Association;2006.

23) Olino TM, McMakin DL, Nicely TA, Forbes EE, Dahl RE, Silk JS. Maternal depression, parenting, and youth depressive symptoms: mediation and moderation in a short-term longitudinal study. J Clin Child Adolesc Psychol 2016;45:279-290.

24) Sowislo JF, Orth U. Does low self-esteem predict depression and anxiety? A meta-analysis of longitudinal studies. Psychol Bull 2013; 139:213-240.

25) Beck AT. Depression: clinical, experimental, and theoretical aspects. Philadelphia: University of Pennsylvania Press;1967.

26) Butler EA, Egloff B, Wilhelm FH, Smith NC, Erickson EA, Gross JJ. The social consequences of expressive suppression. Emotion 2003;3:48-67.

27) Gross JJ. Antecedent- and response-focused emotion regulation: divergent consequences for experience, expression, and physiology. J Pers Soc Psychol 1998;74:224-237.

28) Stikkelbroek Y, Bodden DH, Kleinjan M, Reijnders M, van Baar AL. Adolescent depression and negative life events, the mediating role of cognitive emotion regulation. PLoS One 2016;11:e161062.

29) Birmaher B, Ryan ND, Williamson DE, Brent DA, Kaufman J, Dahl RE, et al. Childhood and adolescent depression: a review of the past 10 years. Part I. J Am Acad Child Adolesc Psychiatry 1996;35:14271439.

30) Kovacs M, Paulauskas S, Gatsonis C, Richards C. Depressive disorders in childhood. III. A longitudinal study of comorbidity with and risk for conduct disorders. J Affect Disord 1988;15:205-217.

31) American Psychiatric Association. Diagnostic and Statistical Manual of Mental Disorders (DSM-5®). 5th ed. Washington, DC: American Psychiatric Pub;2013.

32) Den Hartog HM, Derix MM, Van Bemmel AL, Kremer B, Jolles J. Cognitive functioning in young and middle-aged unmedicated out-patients with major depression: testing the effort and cognitive speed hypotheses. Psychol Med 2003;33:1443-1451. 